\title{
Darkness in the North
}

\author{
Michelle C. Yee, MD, MBA, CCFP*
}

"What time did we give our last epi?" I shouted above the noise, as the respiratory therapist attempted to intubate, and the nurses tried to obtain peripheral access. The alarms rang overhead, and the call for the cardiac arrest was announced. The paramedics who brought him in looked at me with guarded and weary eyes.

I reached down, felt his ice-cold extremities and knew our efforts would be futile. He was rigid diffusely, his extremities contorted in a painful fashion, and his jaw tense and locked in place. The ligature marks were so deeply embedded, I saw them from across the room. He had long been in rigor mortis. There was nothing else that we could do.

I opened up his electronic medical record, five visits in the last 6 months for depression and suicidal ideations.

He was 14 years old - and he was my third adolescent suicidal hanging of the week.

I had been warned that this sometimes occurs in clusters, a "suicide contagion" as it is colloquially called. The impact of one being echoed in the community with shared wounds and pains deeply rooted in history. ${ }^{1}$

This was a new experience for me, working in Northern Ontario. I had read the newspapers, and I knew suicide rates among First Nations youth were five to six times higher than other non-indigenous populations. ${ }^{2}$ Throughout medical school, I had learned about residential schools, forced relocation, and the marginalization of indigenous populations. But I have to admit, I hadn't felt it, until it was in front of my eyes.

"You let him go home?" the charge nurse sternly said to me. He was the father of a young girl from a small reserve community who attempted suicide by a mixed overdose. I nodded my head as the charge nurse explained that it is the hospital policy to have the parent or guardian stay for monitoring the child. "Why would you tell him it's okay to leave?" she asserted.

It was $3 \mathrm{AM}$ and the patient was medically stable. The father had arrived in the afternoon shortly after I called him. He had been there for hours, with one of his other daughters sitting on the floor in the department because we couldn't find any chairs left.

"I don't understand what else I can do," he said. She was admitted earlier this month with a similar overdose attempt. "They started her on medications and said that she'd get better."

I didn't know what to say.

He explained that he had five children at home and that things started to fall apart after their mother committed suicide last year. His eldest ran off, his other son was incarcerated, and his three other children, including my 12-year-old patient, had started using drugs and alcohol daily.

He had asked if he could leave, to go home and make arrangements for his other children because he knew that this daughter would be admitted for a few days. I felt that that was appropriate.

In the intensive care unit (ICU), I caught a glimpse of the possible future of some of these children, the one in which IV drugs and alcohol act as the only solace to numbing the pain. Prolonged intubations for pancreatic and liver failure, end stage nephropathy and bilateral amputations, infective endocarditis and necrotizing fasciitis, the rare zebras I had only seen previously in textbooks.

On my weeks of ICU, I felt more like the angel of death than I did a doctor. With every family meeting, I would meet large groups of individuals - the siblings, aunts, uncles, and the elders. I would always have to clarify who was the substitute decision-maker because it was often a group effort as to who raised the individual, and not necessarily the parents. Oftentimes, I was left

From the *Northern Ontario School of Medicine, Thunder Bay Regional Health Sciences Centre, Department of Emergency Medicine, Thunder Bay, ON

Correspondence to: Dr. Michelle Yee, Northern Ontario School of Medicine, Thunder Bay Regional Health Sciences Centre, Department of Emergency Medicine, 980 Oliver Rd., Thunder Bay, ON P7B 6V4; Email: michellecyee@gmail.com 
explaining that nothing more could be done and that palliation would likely be the best approach. Though their culture is built on stoicism and few words, you could always feel how soul-crushing those words were.

Each mourning ritual was unique. If the families wished to withdraw life support, entire communities would come together to grieve. Some would request priests to be present. Others had elders or family leaders who would guide the prayers and ceremonies; and children, whether they be siblings, nieces, or nephews would be present too. A tragic exposure that at such a young age, they would too frequently become accustomed to.

"This is the fourth time this month we've been in the hospital to witness a death," one of the brothers explained. "It's been a hard month."

I stood next to him in silence, as we watched his loved one take his last few breaths.

In the North, there is a lot of darkness, inundated with the consequences of our cultural past. Intergenerational trauma, abuse, and mistrust are the rule and not the exception. As Maslow's hierarchy dictates, the capacity to rise above the calamity becomes contested if we lack the basic necessities of life. It should not be a privilege to have clean running water, heat, and shelter. It should not be an honour to have access to healthcare and education.

In the North, we look for glimmers of light, but it falls only amongst the shadows.

As physicians, we can only strive to do our best, but it hurts to realize, that sometimes, our best is not enough.

Keywords: Indigenous health, aboriginal health, mental health, suicide, rural medicine

Competing interests: None declared.

\section{REFERENCES}

1. Centre for Suicide Prevention. Toolkit: indigenous suicide prevention. Canada. Available at: https://www.suicideinfo. $\mathrm{ca}$ /resource/indigenous-suicide-prevention/ (accessed 1 March 2018).

2. Government of Canada. Acting on what we know: preventing youth suicide in First Nations. Canada. Available at: https://www.canada.ca/content/dam/hc-sc/migration/hc-sc/ fniah-spnia/alt_formats/fnihb-dgspni/pdf/pubs/suicide/prev_ youth-jeunes-eng.pdf (accessed 4 March 2018). 